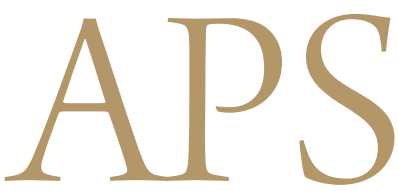

Archives of Plastic Surgery

\title{
Correction of Short Nose Deformity Using a Septal Extension Graft Combined with a Derotation Graft
}

\author{
Moo Hyun Paik, Lo Shui Chu \\ 101 Plastic Surgery Clinic, Seoul, Korea
}

In patients having a short nose with a short septal length and/or severe columellar retraction, a septal extension graft is a good solution, as it allows the dome to move caudally and pushes down the columellar base. Fixing the medial crura of the alar cartilages to a septal extension graft leads to an uncomfortably rigid nasal tip and columella, and results in unnatural facial animation. Further, because of the relatively small and weak septal cartilage in the East Asian population, undercorrection of a short nose is not uncommon. To overcome these shortcomings, we have used the septal extension graft combined with a derotation graft. Among 113 patients who underwent the combined procedure, 82 patients had a short nose deformity alone; the remaining 31 patients had a short nose with columellar retraction. Thirty-two patients complained of nasal tip stiffness caused by a septal extension graft from previous operations. In addition to the septal extension graft, a derotation graft was used for bridging the gap between the alar cartilages and the septal extension graft for tip lengthening. Satisfactory results were obtained in $102(90 \%)$ patients. Eleven (10\%) patients required revision surgery. This combination method is a good surgical option for patients who have a short nose with small septal cartilages and do not have sufficient cartilage for tip lengthening by using a septal extension graft alone. It can also overcome the postoperative nasal tip rigidity of a septal extension graft.

Keywords Nasal cartilages / Ear cartilage / Nasal septum

\author{
Correspondence: Moo Hyun Paik \\ 101 Plastic Surgery Clinic, \\ 4 Apgujeong-ro 71-gil, Gangnam-gu, \\ Seoul 135-956, Korea \\ Tel: +82-2-547-3351 \\ Fax: +82-2-547-3358 \\ E-mail:mhpaik305@naver.com
}

No potential conflict of interest relevant to this article was reported.

\section{INTRODUCTION}

Short nose deformity occurs in a considerable number of East Asians, and successful correction of this deformity, without producing an upturned nasal tip, is an important challenge of Asian rhinoplasty.

In Korea, for short nose correction, a septal extension graft is one of the most widely used surgical techniques and is a simple yet robust method that uses solid septal cartilage to effectively fix the released alar cartilage into a more caudal position, along with tip projection and correction of columellar retraction-all in a single procedure [1]. However, when used alone, a septal extension graft has many possible shortcomings. First, the medial crura of the alar cartilage is fixed to the septal extension graft, which leads to an uncomfortably rigid nasal tip and columella, and subsequently results in unnatural facial animation. Second, it is often difficult to harvest a sufficient amount of septal cartilage from the noses of East Asians. Third, in patients with a short 


\section{Fig. 1. Illustration of the combined graft}

Septal extension lengthens the septum, and the derotation graft secures the released alar cartilage into a more caudal position.

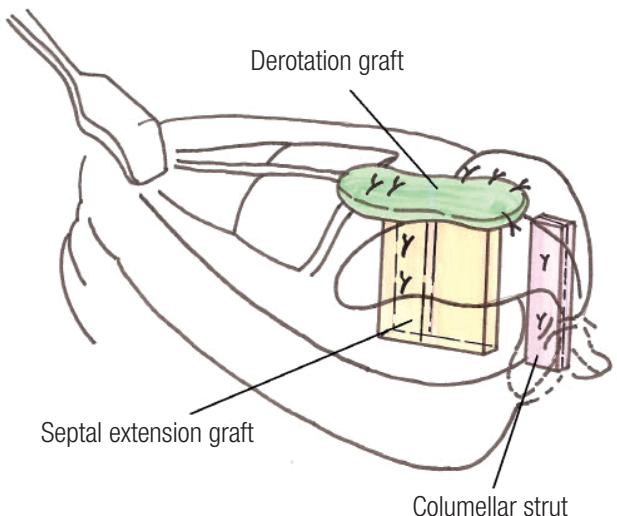

nose and a prior septal extension graft using septal cartilage, a rib cartilage graft is the only viable option for additional tip lengthening as it produces a relatively stiff nasal tip and leaves a chest wall scar [2]. Fourth, a septal extension graft usually gathers the lateral crura to the midline, which, in turn, increases the possibility of a narrow, pinched tip and alar retraction.

Besides the septal extension graft, a derotation graft is a wellknown procedure for short nose correction in Korea and is designed to overcome the abovementioned drawbacks of a septal extension graft [3]. Like a septal extension graft, a derotation graft has limited application when used alone. One of the most typical conditions associated with a short nose is the short septum, which is difficult to correct by using a derotation graft alone and is not sufficiently long for the fixed infrastructure unit to elongate the mobile nasal tip unit.

For the short nose with a relatively short and small septum, we have applied this combination technique to lengthen the short septum by using a septal extension graft and to fix the released alar cartilage into a new caudal position by using a derotation graft in order to preserve as much of the normal anatomy and physiology of the original nasal tip as possible (Fig. 1). We present our experience using this combination procedure to overcome the shortcomings of individual septal extension or derotation graft in the management of short nose deformity.

\section{METHODS}

\section{Patients}

Between December 2003 and March 2013, 113 patients were identified as having undergone the combined septal extension and derotation graft. Of these, 82 patients had short nose deformity; the remaining 31 patients had a short nose compounded by columellar retraction. Of the total number of patients, there were 27 primary and 86 revision cases. In the 86 revision cases, 52 patients had undergone septal extension graft in a previous operation, and among these, 32 patients complained of nasal tip stiffness. Preoperative and postoperative photographs were thoroughly compared for a satisfaction assessment by surgeons and patients. The follow-up ranged from 1 month to 7 years, with a mean of 8.5 months.

\section{Surgical techniques \\ Flap elevation}

An open approach was used in all the cases. In the primary cases, the degloving plane was supraperichondrial from the nasal tip to the cartilaginous vault, and subperiosteal over the nasal bones. In the revision cases with an alloplastic implant and/or adhesion between the scar tissue and skin, a two-layer flap dissection was performed. The first layer was created between the skin and the scar tissue to increase the skin layer release for lengthening. The second layer was formed between the alar cartilage and the scar tissue to allow for caudal rotation of the mobile unit structure. If dorsal augmentation was necessary, the first layer, surrounded by the vascularized tissue, was used for a dermofat graft. For a dorsum implant, the extended second layer beneath the periosteum was utilized along with the previously formed implant pocket.

\section{Caudal release of alar cartilage}

The soft tissue between the alar cartilages and the upper lateral cartilage was released using scissors, leaving the mucosal innerlining intact for a tension-free caudal rotation of the alar cartilages. If adequate caudal rotation could not be achieved, further dissection between the mucosa and the perichondrium beneath the upper lateral cartilages was attempted for additional lengthening. In revision cases with a pre-existing septal extension graft, the medial crura was released from the adherent scar on the membranous septum to allow for caudal movement. In the case of either a severe columellar retraction or an insufficient caudal advancement of the medial crura, a wide dissection was extended on the septal mucosa to facilitate the caudal release of the columella. After all these release procedures, a sufficient release of the alar cartilage was attained without the necessity of a composite graft.

\section{Fixation of caudally released alar cartilage}

A piece of septal cartilage was harvested for the septal extension graft to leave a sufficient L-strut behind, and was secured to the caudal septal margin using either figures-of-eight or mattress sutures with 5-0 nylon. Any pre-existing septal extension graft 
was re-used upon appropriate trimming, deviation correction, and/or extra fixation. In patients with excessive layers of the septal extension graft, the redundant layers were removed to the greatest extent possible to minimize the postoperative nasal tip rigidity without affecting the overall stability. Since a derotation graft usually lowers tip projection, a floating-type columellar strut was fashioned from the double-layered cymba concha, and was used simultaneously to maintain tip projection in all cases except for patients who had a well-developed medical crus. The columellar strut was secured to the medial crura but was not fixed to the septal extension directly. The derotation graft, made of ear cartilage, was fixed to the septal extension and the lateral crura of the alar cartilages in the respective cephalad and caudal locations. The derotation graft was 5 to $10 \mathrm{~mm}$ in width and 15 to $20 \mathrm{~mm}$ in length. If the derotation graft was too weak to support the caudally shifted dome, additional layers of the graft were secured. For strength, both layers of the perichondrium were included in all grafts harvested from the ear. Finally, the graft for tip augmentation was performed as required.

\section{RESULTS}

Of the 113 patients, 102 (90\%) had satisfactory results, with appropriate elongation of the short nose that resembled the preoperative plan according to a photographic assessment by the surgeons and patients. The 32 patients, who had complained of rigid tip secondary to previous septal extension grafts, reported satisfactory improvements with a more pliable and natural nose.

Of the total, 11 patients $(10 \%)$ required a revision procedure because of overcorrection (6 patients) or undercorrection (1 patient) of the nasal length, poor tip projection (2 patients), inadequate correction of columellar retraction (1 patient), and a visible graft on the supratip (1 patient).

In patients with overcorrection, the derotation graft was separated from the alar cartilage and fixed into a new position upon the cephalic rotation of the tip. In contrast, to solve the undercorrection of lengthening, the derotation graft was separated from the alar cartilages and re-secured with the caudal release of alar cartilages from the surrounding structure. If necessary, an

Fig. 2. A case of combined graft

A 62-year-old patient with severe contraction secondary to chronic implant infection. (A) Preoperative front view. (B) Postoperative front view at 1 year. (C) Preoperative lateral view. (D) Postoperative lateral view at 1 year.
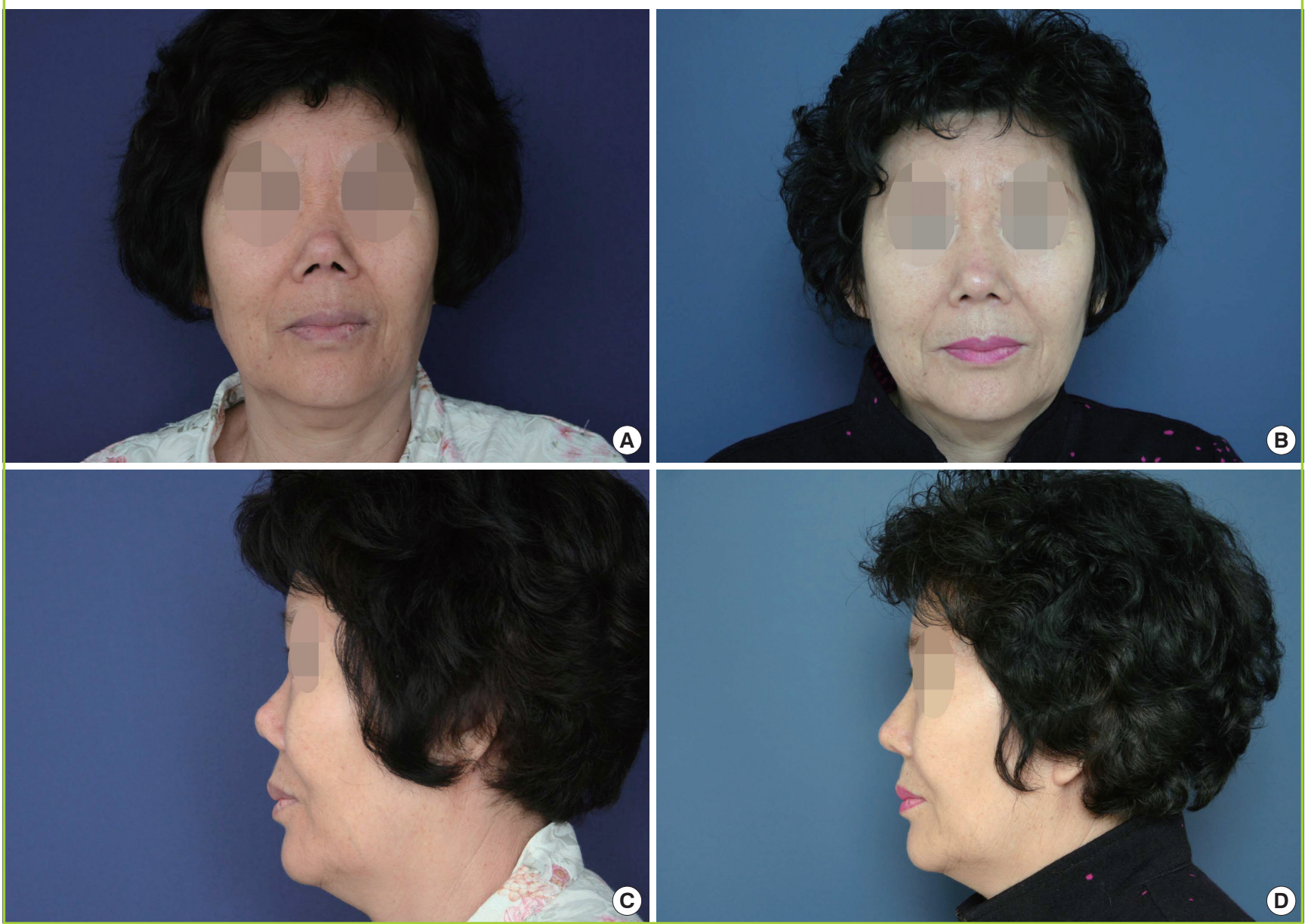
additional derotation graft, shield, or cap graft was performed. For patients with poor tip projection or inadequate correction of columellar retraction, either a cartilage graft or an allogenic/ autogenous dermis graft was added. The visible derotation graft on the supratip was resolved by shaving the graft.

\section{Case 1}

A 62-year-old woman presented with a severely contracted nose and columellar retraction. Previously, the patient had a silicone implant, which was removed at an outside clinic after chronic infection. A two-layer dissection, above and beneath the scar tissue, was performed for skin and alar cartilage elongation. A septal extension graft using septal cartilage was performed, along with a columellar strut, derotation graft, tip onlay, and shield graft harvested from the ear. A dermofat graft was used for dorsal augmentation between the skin and the scar tissue (Fig. 2).

\section{Case 2}

A 41-year-old patient presented with a short nose compounded by a rigid narrow pinched tip and alar retraction. In a prior operation, she had undergone a septal extension graft and silicone implantation. The flap elevation was performed above and beneath the implant capsule. Alar cartilages were released from the septal extension graft and the upper lateral cartilage to allow for the caudal rotation of the nasal tip. In addition to the pre-existing septal extension graft, an alar spreader graft-type derotation graft, umbrella-shaped columellar strut, tip onlay, and shield graft were performed for further elongation and the correction of the pinched narrow tip and alar retraction. From the nasal root to the tip, a dermofat graft was used for dorsal augmentation and coverage of thin skin (Fig. 3).

\section{DISCUSSION}

Structural grafts such as septal extension grafts and derotation grafts can be performed by the use of ear, septal, or rib cartilages. In general, each method has its own most widely used and accepted type of cartilage graft. For example, the most often used

\section{Fig. 3. A case of combined graft}

A 41-year-old patient with prior septal extension and dorsal implantation, complicated by a narrow, pinched tip and persistent short nose deformity. (A) Preoperative front view. (B) Postoperative front view at 1 month. (C) Preoperative lateral view. (D) Postoperative lateral view at 1 month.
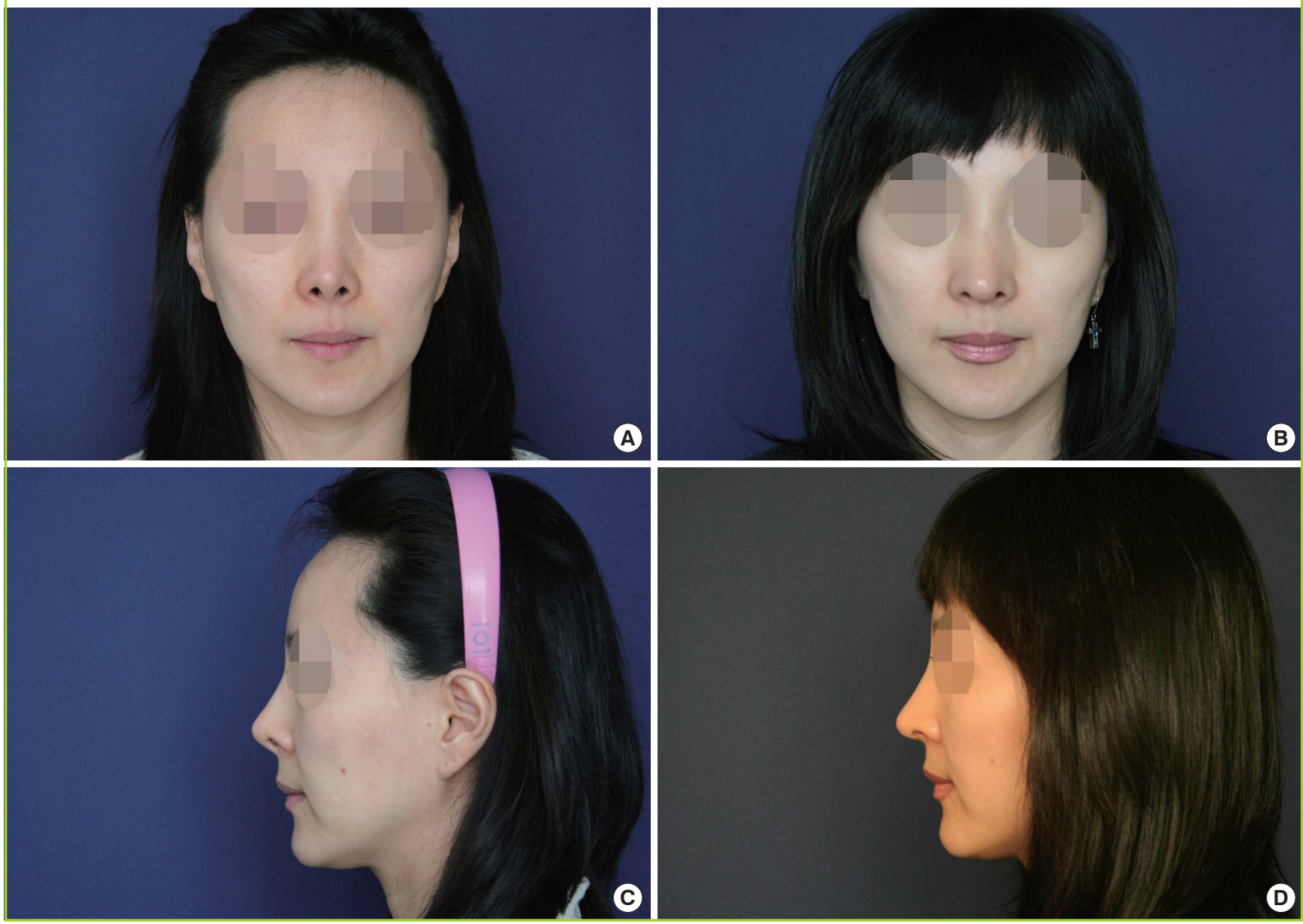


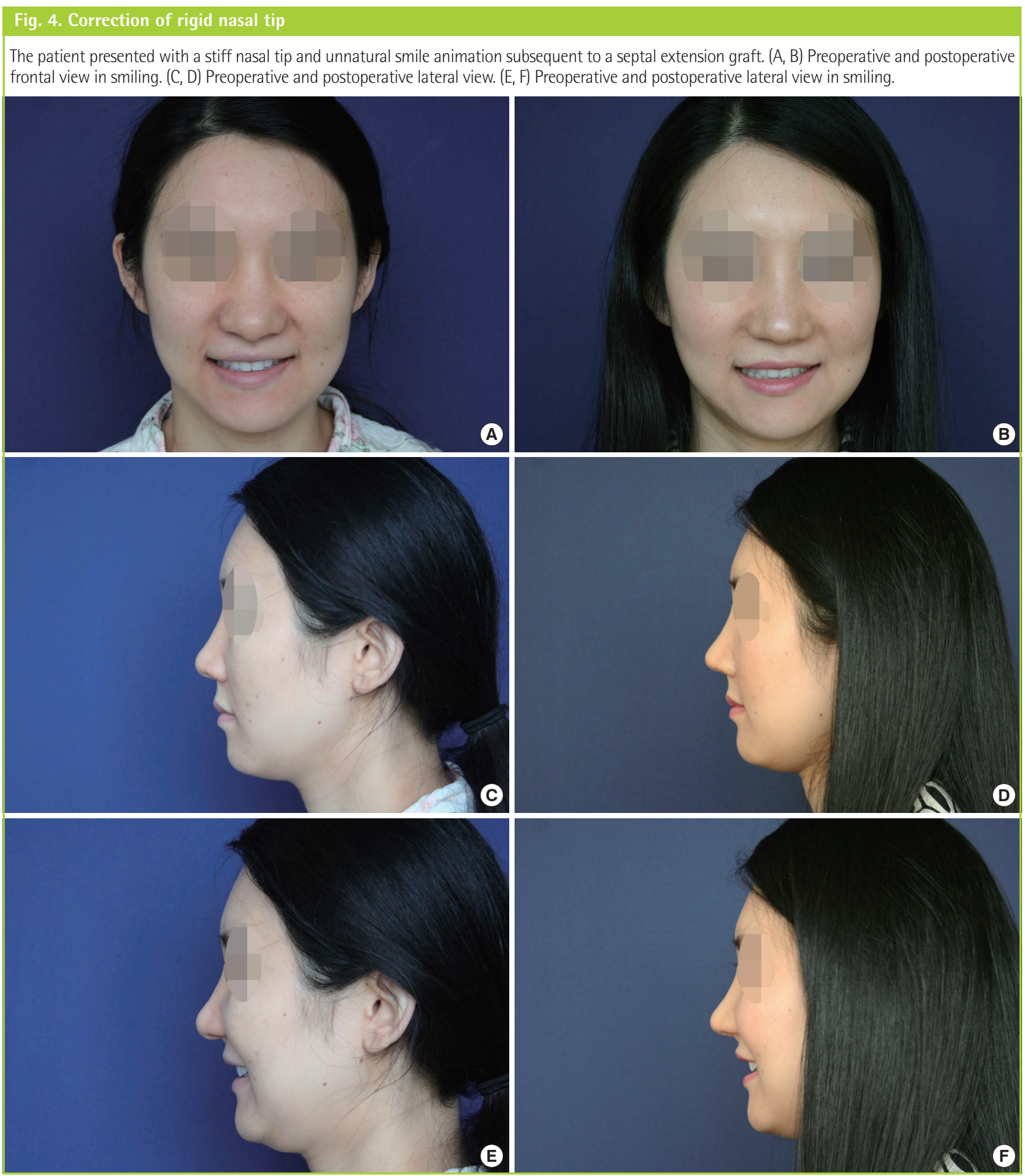

material for a derotation graft is the ear cartilage, whereas the most frequently used material for a septal extension graft is the septal cartilage, with the rib cartilage employed less frequently. With respect to the selection of the graft method and the material for short nose correction, the individual nose and donor site condition must be considered, in order to maintain the physical properties of the natural nose to the maximum possible extent.

The septal extension graft, as first described by Byrd et al. [4], is a technique widely used in rhinoplasty for short nose correction [5]. It is a powerful and straightforward way of lengthening the nose while controlling tip projection and correcting columellar retraction [1]. In this method, abundant septal cartilage 
having adequate strength is essential. However, an insufficient amount and solidity of septal cartilage is not uncommon in the East Asian population. This is especially the case in patients with a short nose, among whom only a small amount of cartilage may be obtained when considering that more of the L-strut should be left behind for stability. This is the main reason why we do not advocate the septal cartilage as a primary choice for cartilage grafts in Asian rhinoplasty. As the frequency of revision rhinoplasty in Korea continues to increase, many surgeons favor the use of rib cartilage as a last resort when no redundant septal cartilage is available because of the previous use of a septal graft [6]. Postoperative rigid tip is a common problem after a septal extension graft using septal cartilage, and the use of rib cartilage further aggravates this problem and leaves the patient with a chest wall scar.

To overcome the disadvantages of septal extension graft, we have devised a procedure called derotation graft, which uses ear cartilage [3]. In the past decade, we have performed more than one thousand derotation grafts. This method makes possible accurate pre/intraoperative estimation of the amount of ear cartilage to be harvested. The flexibility of the ear cartilage graft results in a less rigid nasal tip and avoids interference with the function of the membranous septum.

Although a derotation graft is a very useful way of correcting a short nose, it also has its limitations in application. It is difficult to effectively elongate the nose if the caudal septum is considerably short and/or if the distance between the caudal septal margin and the new alar cartilage position is too large to allow the graft to bridge the gap maintaining the tip elongation. Even if the tip lengthening is sufficient, derotation graft cannot resist the tension after closure in patients with tight skin and a short septal length. A derotation graft always lowers the tip and is commonly used along with a columellar strut, and the latter can also correct mild degrees of columellar retraction. However, for patients who have a short nose and severe columellar retraction, a septal extension graft is necessary to push down the columellar base appropriately.

To overcome the limitation of a septal extension and derotation graft, the combination technique that we suggest is a powerful and reasonable procedure for short nose correction in the population discussed. The basic concept underlying this method is that septal extension graft is used for the sole purpose of lengthening the short septum and derotation graft is reserved for caudal rotation of alar cartilages. If the amount and strength of septal cartilage for septal extension is not sufficient, derotation allows further tip lengthening, without resorting to the use of rib cartilage. In patients who have undergone a prior septal extension graft without adequate elongation of the short nose, the derotation graft can complement the septal extension graft. With this hybrid method, we were able to remarkably reduce the use of rib cartilage graft and, consequently, avoid chest scarring and deteriorating nasal tip stiffness. A derotation graft and columellar strut also provide many benefits for tip-plasty. The purpose of the columellar strut is to augment the nasal tip, since a derotation graft deprojects the tip and pushes out the columellar base in cases in which the septal extension graft cannot sufficiently correct columellar retraction. Where a narrow tip or nostril pinching is demonstrated or predicted to be at high risk, an umbrella-shaped columellar strut was utilized to widen the dome of the nasal tip. In the presence of either alar retraction or narrow lateral crural width at a high risk of pinched tip deformity after approximation of the lateral crura, a derotation graft was interposed between the cephalic margins of the two lateral crura. At this point, the degree of spreading could be controlled by the width of the derotation graft. The distance between the two alar cartilages cannot be directly controlled by a septal extension graft alone because it always approximates the alar cartilage towards the midline.

One of the reasons why we prefer this combination technique is the preservation of tip mobility, which many surgeons too frequently ignore. The human nose is quite an amazing structure, with complex anatomy and physical properties. From a histological point of view, septal and rib cartilages are composed of hyaline cartilage, while ear cartilage is composed of elastic cartilage [7]. Structurally, the nose can be divided into three units according to the distinctive physical characteristics of each. The least projecting portion of the nose is the upper dorsum, whose main component is solid, immobile bone. The cartilaginous septum and the upper lateral cartilages form the stiff lower dorsum, with limited side-to-side flexibility. The most projecting nasal tip is composed of alar cartilages, which are highly flexible elastic cartilages. Consequently, the most projecting parts in the human face, ears, and nose are composed of elastic cartilage, and their great omnidirectional flexibility is protective of these appendages against external forces.

The basic concept of short nose correction is to use the fixed unit as a base to secure the mobile unit, the alar cartilages, into a new caudal position with a graft between these two units. As such, any possible means to correct a short nose would result in a variable degree of tip rigidity. In our experience, a septal extension using hyaline cartilage such as septal or rib cartilage causes the nasal tip to be much less pliable than a derotation graft using elastic ear cartilage would allow. A septal extension is a verticaltype, stiff hyaline cartilage graft through the membranous septum; it fixes the mobile alar cartilages directly, including the medial crura, as a single unit, resulting in a rigid postoperative 
nasal tip. Although some degree of side-to-side mobility can be preserved, the resulting tip is hardly flexible in the cephalic direction. In contrast, a derotation graft uses the flexible ear cartilage as a cushion buffer through the horizontal plane, does not obliterate the membranous septum, and allows the nasal tip to move as much as possible in all directions. Therefore, we have used this combination procedure to minimize the loss of tip pliability when the septal extension graft alone could not appropriately address the problems of a short nose. Although not included in this study, satisfactory outcomes could be obtained in revision patients who presented with tip rigidity and adequate nasal length by adequately resecting a caudal portion of the preexisting septal extension graft with a hybrid conversion to this method (Fig. 4).

Furthermore, if caudal septal deviation were observed using septal extension graft alone in a patient, the nasal tip or columella was more likely to be tilted without precise correction of the deviated caudal septum because the alar cartilages were fixed directly to the extended septum. In our method, the tip is projected by the columellar strut and rotated by the derotation graft in a mechanically separate manner, without any direct forcebearing fixation between the alar cartilage and the extended septum. Therefore, the secondary tip and columellar deviation from a primary deviation of the caudal septum is less likely to occur.

The septal extension graft combined with the derotation graft has many benefits in the management of a short nose. It is a ro- bust lengthening technique that re-creates a nose that is not only aesthetically pleasing but also has a more pliable postoperative nasal tip, particularly in East Asian patients.

\section{REFERENCES}

1. Hobar PC, Adams WP, Mitchell CA. Lengthening the short nose. In: Gruber RP, Stepnick D, editors. Rhinoplasty: current concepts. Philadelphia, PA: Saunders Elsevier; 2010. p. 327-33.

2. Toriumi DM, Swartout B. Asian rhinoplasty. Facial Plast Surg Clin North Am 2007; 15:293-307.

3. Paik MH, Chu LS. Correction of the short nose using derotation graft. Arch Aesthetic Plast Surg 2012;18:35-44.

4. Byrd HS, Andochick S, Copit S, et al. Septal extension grafts: a method of controlling tip projection shape. Plast Reconstr Surg 1997;100:999-1010.

5. Ha RY, Byrd HS. Septal extension grafts revisited: 6-year experience in controlling nasal tip projection and shape. Plast Reconstr Surg 2003;112:1929-35.

6. Cochran CS, Gunter JP. Secondary rhinoplasty via the external approach. In: Azizzadeh B, Murphy MR, Johnson Jr CM, et al., editors. Master techniques in rhinoplasty. Philadelphia, PA: Saunders Elsevier; 2011. p. 91-105.

7. Liu W, Cao Y. Repari, grafting, and engineering of cartilage. In: Neligan PC, Gunter GC, editors. Plastic surgery: principles. Philadelphia, PA: Saunders Elsevier; 2012. p. 397-424. 